\title{
Bilateral Optic Neuropathy by Methanol Poisoning
}

\section{S Amrani Joutei*, A Haddad, N Mataa, L El Maaloum, B Allali and A El Kettani}

Ophthalmology Department, Hospital August 20, Hassan II, CHU Ibn Rochd

Casablanca, Morocco

*Corresponding Author: S Amrani Joutei, Ophthalmology Department, Hospital August 20, Hassan II, CHU Ibn Rochd Casablanca, Morocco.
Received: June 11, 2021

Published: October 06, 2021

(C) All rights are reserved by $\mathbf{S}$ Amrani Joutei., et al.

\begin{abstract}
Methanol poisoning optic neuropathy is a rare but serious condition that can cause bilateral blindness. It can occur after a deliberate or accidental ingestion of this toxic.

We report the case of methanol poisoning whose evolution was marked by the installation of bilateral optic atrophy despite treatment combining corticosteroid therapy and vitamin B.

Through this observation we recall the physiopathology as well as the clinical, therapeutic and evolutionary aspects of optic neuropathy by methanol intoxication.
\end{abstract}

Keywords: Bilateral Optic Neuropathy; Methanol Poisoning

\section{Introduction}

The Methanol intoxication can lead an optic atrophy, which often has a poor prognosis. The toxicity is not in the methanol but it is in one of its metabolites, notably formic acid which has a neurotoxic effects. The evolution depend on the severity of the intoxication and efficacity of the treatment. There are maybe a complete cure which is rare, incomplete regression or permanent blindness from optic atrophy. We report a case of methanol blinding optic neuropathy.

\section{Observation}

We report the case of a male of 30 years old, who consulted in the ophthalmology emergency the third day for bilateral blindness occurring 24 hours after ingestion of an alcoholic mixture. The clinical examination of the patient found a polypnea with sweat, and it was admitted in intensive care. The ophthalmologic examination revealed an absence of bilateral light perception with an a reactive mydriasis (Figure 1). The Fundus examination notes mild optic disc hyperemia and severe nerve fiber swelling with retinal edema were visible in both eyes (Figure 2). The biological assessment shows a metabolic acidosis corrected by bicarbonates. Cranio-orbital MRI is normal. On the electro-physiological level, the visual evoked flash potentials are extinguished. We referred the patient to the anti-poison and pharmacovigilance center at Rabat city for toxicological analysis which objective $7.8 \mathrm{~g} / \mathrm{l}$ of Ethanol and 848 $\mathrm{g} / \mathrm{l}$ of Methanol in the product ingested and no trace on the blood sample and urine (explained by the delay of blood sampling). The patient benefits from a bolus treatment of methylprednisolone at 1 $\mathrm{g} / \mathrm{d}$ for 3 days combined with vitamin B at $1 \mathrm{~g} / \mathrm{d}$ intramuscularly. No improvement was noted during follow-up. Two months later, 
The visual acuity was stationary at negative light perception with bilateral optic atrophy.

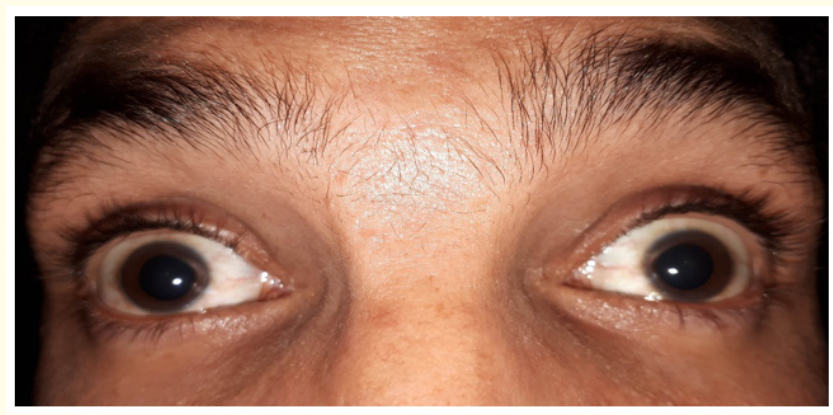

Figure 1: Bilateral areactive mydriasis.

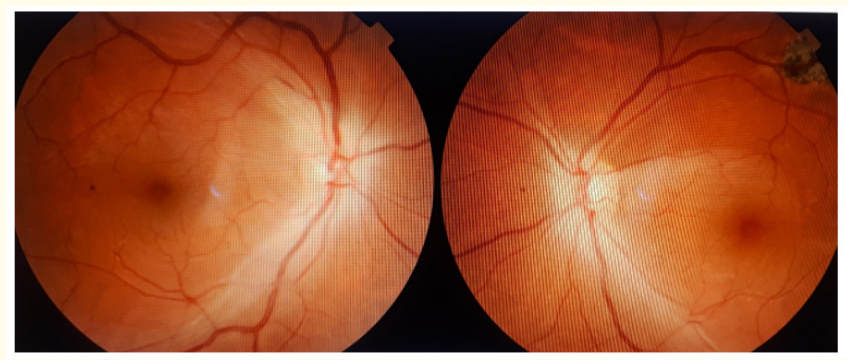

Figure 2: Fundus examination: mild optic disc hyperemia and severe nerve fiber swelling with retinal edema visible in both eyes.

\section{Discussion}

Methyl alcohol or methanol is a synthetic alcohol found in denatured alcohol and in many household products such as methylated spirits, solvents and thinners, dyes and tinctures. Methanol poisoning most often occurs after ingestion, accidental or for suicidal purposes, more rarely by contact or inhalation of vapors. The minimum lethal dose is usually $30 \mathrm{ml}$ with individual susceptibilités. Methanol is metabolized in the liver and is transformed under the action of the enzyme alcohol-dehydrogenase into formaldehyde, then into formic acid which is the toxic metabolite of methanol and inhibits the cytochrome oxidase complex of the mitochondrial respiratory chain, which causes cellular anoxia in the retina and optic nerve. Two phenomena ensue: edema compressing the axons of the optic nerve which causes axoplasmic stasis responsible for papillary edema, and a direct impairment of nerve conduction. Clinically, signs of methanol poisoning appear 6 to 12 hours after ingestion and depend on the dose ingested. The minor form is characterized by the presence of isolated digestive symptoms such as nausea and vomiting. Upon ingestion of larger doses, ocular and neuropsychiatric signs appear, ranging in severe form to coma and death. Ocular symptoms begin between $30 \mathrm{~min}$ and 72 hours after ingestion as visual fog, phosphenes and rapid and major visual loss. The fundus shows papillary hyperemia followed by edema of the papilla and the adjacent retina. Initial mydriasis has a poor prognosis. The visual evoked potentials are very altered, even extinct. The electroretinogram shows a decrease in the amplitude of the $b$ wave due to damage to the photoreceptors. The CT scan can show lesions characteristic of methanol poisoning in the form of symmetrical hypodensities of the putaminal regions. The course depends on the severity of the poisoning and how long it takes to start treatment. In severe poisoning, the subject dies from respiratory distress. Complete recovery is rare. Most often the visual prognosis is poor, with severe and definitive visual decline by optic atrophy. In our patient, the major visual loss associated with bilateral mydriasis is a poor prognosis and testifies to the severity of the intoxication, which explains the lack of visual recovery. Treatment should be started in the first hours after ingestion. It aims to control the metabolic balance by ingestion of bicarbonate, the elimination of the toxic agent by hemodialysis and the inhibition of hepatic degradation by alcohol-dehydrogenase inhibitors such as ethyl alcohol and 4- methyl-pyrazole or fomepizole, the effectiveness of which has been proven by a prospective multicenter study which has shown the utility of this treatment against metabolic acidosis, optic neuropathy and neurological impairment. Some authors suggest corticosteroid therapy as a bolus or orally at a rate of $1 \mathrm{mg} / \mathrm{kg} /$ day for one month to reduce papillary edema. Rotenstreich reports a case of complete recovery of visual acuity after corticosteroid treatment initiated 2 weeks after methanol intoxication. We also recommended this treatment in our patient, but we did not notice any visual recovery [1-6].

\section{Conclusion}

Despite the scarcity of this optic neuropathy, the severity of the prognosis and the necessity of a fast treatment requires a preco- 
cious diagnosis, in order to establish a specific treatment by dehydrogenase alcohol inhibitors, allowing an improvement of the functional and vital prognosis.

\section{Bibliography}

1. Masson E. "Neuropathie optique bilatérale par intoxication aiguë au méthanol. EM-Consulte (2020).

2. Masson E. "Neuropathies optiques médicamenteuses, toxiques et carentielles. EM-Consulte (2020).

3. Cursiefen $\mathrm{C}$ and Bergua A. "Acute bilateral blindness caused by accidental methanol intoxication during fire "eating". British Journal of Ophthalmology 86.9 (2002): 1064-1065.

4. Sharma M., et al. "Methanol-induced optic nerve cupping". Archives of Ophthalmology 117.2 (1999): 286.

5. Oeil et pathologie générale. Unithèque (2020).

6. Essama Mbia J-J., et al. "Fomepizole therapy for reversal of visual impairment after methanol poisoning: a case documented by visual evoked potentials investigation". American Journal of Ophthalmology 134.6 (2002): 914-916.

Volume 4 Issue 11 November 2021

(C) All rights are reserved by $S$ Amrani Joutei., et al. 